Minireview

\title{
Estimating rates and patterns of morphological evolution from phylogenies: lessons in limb lability from Australian Lerista lizards John J Wiens
}

Address: Department of Ecology and Evolution, Stony Brook University, Stony Brook, NY 11794-5245, USA. Email: wiensj@life.bio.sunysb.edu

Published: 24 February 2009

Journal of Biology 2009, 8:19 (doi:10.1 186/jbioll I2)

The electronic version of this article is the complete one and can be found online at http://jbiol.com/content/8/2/19

(C) 2009 BioMed Central Ltd

\begin{abstract}
Squamates (lizards and snakes) offer an exciting model system for research on the evolution of body form. A new phylogenetic study in BMC Evolutionary Biology of Australian lizards shows remarkable evolutionary lability in digit numbers among closely related species, but also highlights important challenges in this area.
\end{abstract}

A major goal in evolutionary biology is to explain the great phenotypic diversity of life. Why are there organisms that look as varied as octopi, redwoods, earthworms, seagulls, centipedes, jellyfish, and Scarlett Johansson? But in many cases, understanding major changes in body form is difficult because such dramatic changes have happened only rarely and mostly in the distant past (for example, the remarkable shell of turtles arose only once, and more than 200 million years ago).

Enter the squamates (lizards and snakes). Within squamates, one of the most dramatic changes in body form in animals has occurred: from a four-limbed lizard-like body plan to an elongate, limbless snake-like one. Although everyone is familiar with snakes, a similar transition may have occurred dozens of times and across many different clades [1,2], sometimes among species of the same genus [1]. As a model system for macroevolutionary research, squamates offer both natural replication and controls (that is, repeated changes in body form, sometimes among closely related species).

Recent studies within squamates have begun to utilize molecular phylogenies to reveal the patterns, rates, and tempo of these dramatic changes in body form [2-5]. Because phylogeny is essential to understanding the "what happened' of evolution, these analyses represent the first steps towards an integrative understanding of major transitions in squamate body form. Molecular phylogenies are particularly important, because phylogenetic analyses of morphological data may be confounded by these dramatic changes in body form [6], whereas molecular characters should generally be unaffected.

Among all the many clades in which body form could be studied, the Australian lizard genus Lerista may be the most interesting. Lerista is one of only a few genera in which the full range of these body forms is represented [1], from a lizard-like body with well-developed limbs with five digits (Figure 1a), to fully limbless forms with no external limbs or digits (Figure 1c), with many seemingly intermediate morphologies as well (for example, species with one to four digits on a limb; Figure 1b). Past studies $[7,8]$ have described morphological variation in Lerista, but without a phylogeny this variation is difficult to interpret evolutionarily.

Skinner et al. [9], in a study published in BMC Evolutionary Biology, have now provided an extensive molecular phylogeny 


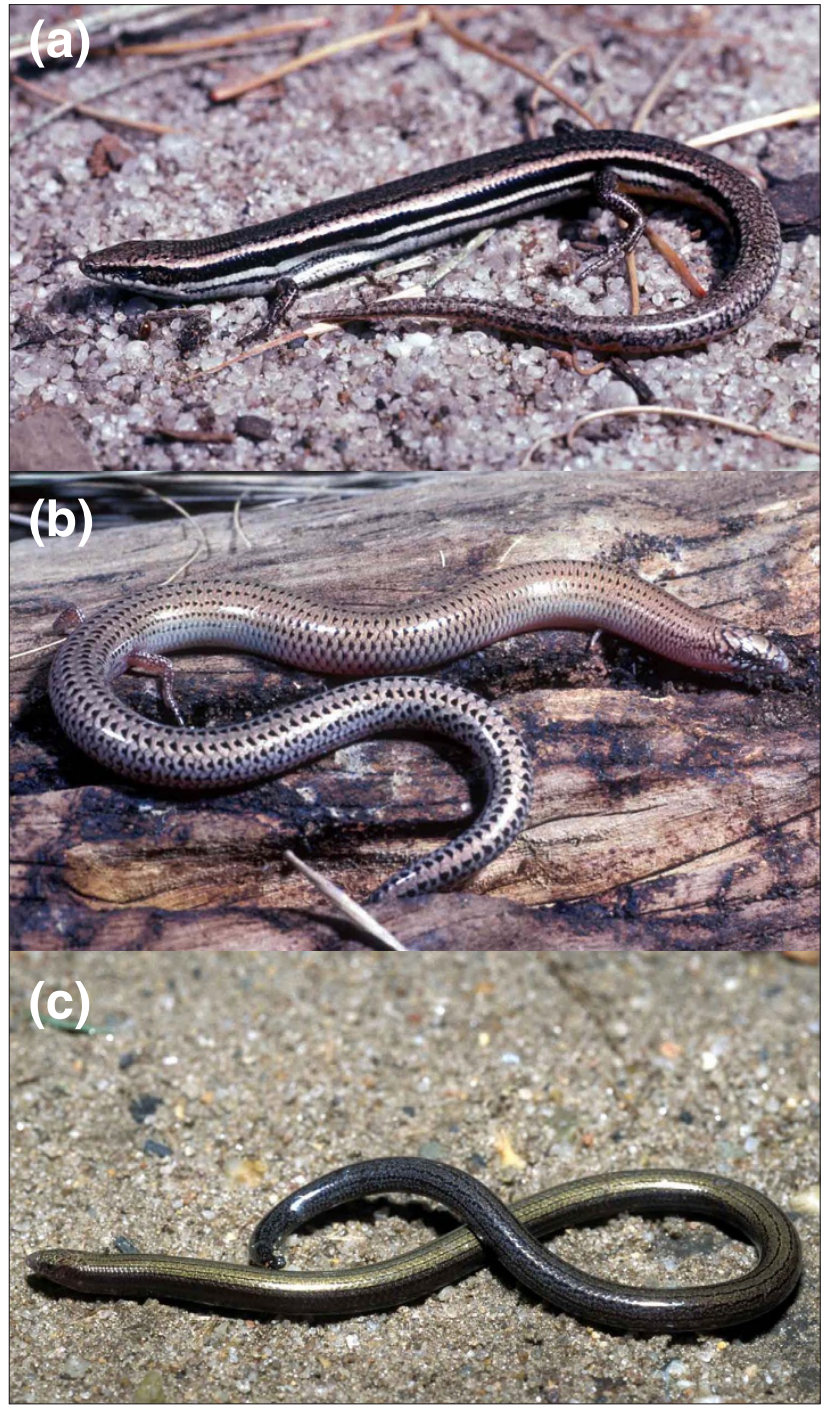

Figure I

Body form variation in Lerista. Three species of the Australian skink genus Lerista are shown, illustrating the remarkable morphological changes that have occurred within this genus, as elucidated in the new paper by Skinner et al. [9]. (a) The species at the top (Lerista microtis) has the typical lizard-like body form with five digits per limb, (b) the species in the middle (Lerista punctovittata) has an intermediate body form with reduced limbs whereas (c) the species at the bottom (Lerista ameles) has the elongate snake-like body form and lacks external limbs and digits. The photos of $L$. microtis and $L$. punctovittata are by Mark Hutchinson and the photo of $L$. ameles is by Marco Sacchi.

for Lerista, which they use to examine the evolution of digit numbers. They have found some exciting results, but their study also illustrates some important methodological challenges. These challenges are not unique to the Lerista study, but may affect many phylogeny-based studies of character evolution. Here, I will briefly review what we have learned from recent phylogenetic analyses of squamate body-form evolution, and especially the new study of Lerista.

Past studies [2-4] have combined phylogenies with morphometric and ecological data to show that there are really two types of snake-like squamates. One is the long-tailed surface dweller, in which much of the overall body length is tail, and which often lives in dense grass. The other is the shorttailed burrowing morph, which has a short tail, elongate trunk, and typically lives underground. The long-tailed morph has evolved five times, whereas the short-tailed burrower has evolved at least twenty times. However, many more origins of the burrowing morph remain to be documented among the 'skinks' (the common name for lizards of the family Scincidae), given that skinks include many limb-reduced taxa not yet included in phylogenies [2].

Kohlsdorf and Wagner [5] analyzed phylogeny and digit evolution in the South American lizard genus Bachia and found strong evidence that lost digits can be evolutionarily regained. This is the sort of pattern that only a phylogeny can reveal. However, a recent simulation study has raised the possibility that some inferred reversals might be artifacts of some likelihood-based reconstruction methods [10].

Brandley et al. [4] analyzed body form transitions across all squamates (258 species sampled) using a phylogeny in which the lengths of the branches reflect time (inferred using modified molecular clock methods [11]). They found that complete loss of external limbs seemed to require 16 million years or more, but might be far more rapid. They also showed that intermediate morphologies (for example, some digits lost but not all) persist for long periods of time, in some cases longer than the time needed for the transition from fully limbed to fully limbless morphologies. This result suggests that these seemingly intermediate morphologies are not simply temporary transitions between extremes. They also found some cases where lost digits seemed to re-evolve.

The new study by Skinner et al. [9] has reconstructed the phylogeny of the pivotal skink genus Lerista, sampling 79\% of the 91 described species [12]. They also reconstructed digit numbers (but not body form or limb loss) on their tree using the statistical method of maximum likelihood. Their results show remarkable evolutionary lability in digit numbers. For example, they found clades of species with five digits adjacent to those with none, species with no digits (on the forelimb, or manus) at the base of the tree, species with four digits deeply nested among species with more extreme reduction (none to two), and seemingly four separate instances of loss of all digits on both limbs. They also used molecular-clock based time estimates to infer rates of digit change, and claimed that transitions from five to no 
digits can occur in 3.6 million years or less, much faster than rates estimated by Brandley et al. [4] for other clades. Although one might have predicted more rapid digit loss in Lerista (they are congeners), the extreme lability of digit evolution revealed by this new phylogeny is quite exciting.

However, this same lability may make it challenging to reconstruct precisely what happened and how quickly. Their reconstructions suggest multiple losses of digits from the manus (hand) with no reversals (as expected), but they found a very different pattern for the pes (foot). Using the statistically best-fitting likelihood model for their pes data, they found that digits were regained at a higher rate than the rate at which digits were lost, implying that reacquisition of lost digits is common (a remarkable result). Nevertheless, they prefer reconstructions in which there are no reversals. They argue that reconstructions with reversals are implausible because some reconstructed ancestors have digital patterns not seen in extant Lerista (for example, more digits on the manus than pes). However, similar combinations do occur in closely related taxa (for example, Anomalopus mackayi has two digits on the forelimb and one on the hindlimb [4]), and still occur under their favored model, only less frequently. They also argue that the fit of models of evolution in which reversals occur are not significantly different from those in which they do not. Given the dramatic sensitivity of the results to models that have statistically similar fit to the data, it appears that the reconstructed patterns and rates of digit loss and gain are actually quite uncertain, especially for the pes. Previous authors have suggested that rapid rates of character change may obscure past evolutionary history and lead to uncertainty in reconstructions [13], and they may create ambiguity in selecting models as well. Furthermore, the issue of character-state ordering (for example, is a change from two digits to one treated as more likely than a change from five to one?) was not addressed, but might also be critical in accurately reconstructing the evolution of characters that have multiple states, like digit number [4].

The inference of rates of change also requires some additional caution. Inferring absolute rates of change from a phylogeny requires reconstructing states on a tree with estimated ages for each branch. Molecular analyses of divergence times generally require calibration of clade ages using one or more fossil taxa [11]. But these fossils can generally only constrain the minimum age of a clade; unsampled fossils can always show the clade to be older [11]. Because no Lerista fossils are available, these authors used fossils from a clade of distantly related skink taxa, but did not address the possibility that this clade might be older than indicated by the few available fossils. Coincidentally, Brandley et al. [4] estimated ages of comparable Lerista species using similar methods [11], but found them to be nearly twice as old. If Lerista was twice as old, this would mean that the rates of evolutionary change were actually much slower. Clearly, there is considerable uncertainty in estimating these ages, which may influence estimates of rates.

Estimates of evolutionary rates can also be sensitive to character reconstructions. If the ancestral reconstructions are ambiguous (as may be the case in Lerista), then it may be difficult to say what the changes are, where they occur on the tree, and how long they took to occur. Given that Lerista is not extremely old and contains the full range of digit numbers, there must have been rapid change. But being more precise than this requires precise estimates of ages and ancestral states. Perhaps the best approach for future phylogeny-based studies of character evolution would be to confront this uncertainty head on, and present a range of estimated rates across different dates and reconstruction models.

In summary, Skinner et al. [9] provide an invaluable phylogeny for one of the most interesting groups of lizards for the analysis of digit and body form evolution. But paradoxically, Lerista may also prove to be quite difficult to study, as character reconstructions seem ambiguous, rendering precise inferences about rates or patterns of change somewhat challenging. The rapid rate of digit evolution may be primarily responsible for this ambiguity, and similar ambiguities may plague other studies of rapidly evolving characters.

Phylogenetic studies are only the beginning of integrative approaches to understanding evolution of body form in living taxa. In addition to inferring the 'what happened' and 'how quickly', we also want to know the 'why' (for example, functional consequences of different body forms) and the 'how' (for example, what genetic and developmental mechanisms explain digit loss, limb reduction, and body elongation). The phylogeny is not the final answer, but an important initial step and a crucial framework for all future evolutionary studies.

\section{References}

I. Lande R: Evolutionary mechanisms of limb loss in tetrapods. Evolution 1978, 32:73-92.

2. Wiens JJ, Brandley MC, Reeder TW: Why does a trait evolve multiple times within a clade? Repeated evolution of snake-like body form in squamate reptiles. Evolution 2006, 60:123-141.

3. Wiens JJ, Slingluff JL: How lizards turn into snakes: a phylogenetic analysis of body-form evolution in anguid lizards. Evolution 200I, 55:2303-2318.

4. Brandley MC, Huelsenbeck JP, Wiens J]: Rates and patterns in the evolution of snake-like body form in squamate reptiles: evidence for repeated re-evolution of lost digits and long-term persistence of intermediate body forms. Evolution 2008, 62:2042-2064.

5. Kohlsdorf T, Wagner GP: Evidence for the reversibility of digit loss: a phylogenetic study of limb evolution in the genus Bachia (Gymnophthalmidae: Squamata). Evolution 2006, 60:1896-1912. 
6. Lee MSY: Convergent evolution and character correlation in burrowing reptiles: towards a resolution of squamate relationships. Biol J Linn Soc 1998, 65:369-453.

7. Greer AE: Limb reduction in the genus Lerista. I. Variation in the number of phalanges and presacral vertebrae. J Herpetol 1987, 2I:267-276.

8. Greer AE: Limb reduction in the genus Lerista. 2. Variation in the bone complement of the front and rear limbs and the number of postsacral vertebrae. J Herpetol 1990, 2I:142-I50.
9. Skinner A, Lee MSY, Hutchinson MN: Rapid and repeated limb loss in a clade of scincid lizards. BMC Evol Biol 2008, 8:3 I0.

10. Goldberg EE, Igic B: On phylogenetic tests of irreversible evolution. Evolution 2008, 62:2727-274I.

II. Sanderson MJ: Estimating absolute rates of molecular evolution and divergence times: a penalized likelihood approach. $\mathrm{Mol}$ Biol Evol 2002, 19:101-109.

12. The TIGR Reptile Database [http://www.reptile-database.org/]

13. Schluter D, Price TD, Mooers $A \varnothing$, and D. Ludwig D: Likelihood of ancestor states in adaptive radiation. Evolution 1997, 51:1699-I7I I. 